\title{
Light quark masses, chiral condensate and quark-gluon condensate in quenched lattice QCD with exact chiral symmetry
}

\author{
Ting-Wai Chiu, Tung-Han Hsieh
}

Department of Physics, National Taiwan University, Taipei, Taiwan 106, Taiwan ROC

Received 4 June 2003; received in revised form 9 September 2003; accepted 16 September 2003

\begin{abstract}
We determine several quantities in quenched lattice QCD with exact chiral symmetry. For 100 gauge configurations generated with Wilson gauge action at $\beta=6.0$ on the $16^{3} \times 32$ lattice, we compute quenched quark propagators for 13 bare quark masses. The pion decay constant is extracted from the pion propagator, and from which the inverse lattice spacing is determined to be $a^{-1}=1.979(6) \mathrm{GeV}$. The parameters $(\delta, A, B)$ in the pseudoscalar meson mass formula in quenched chiral perturbation theory $(\mathrm{q} \chi \mathrm{PT})$ to one-loop order are determined. Further, we measure the index (topological) susceptibility of these 100 gauge configurations, $\chi_{t}=(175 \pm 6 \mathrm{MeV})^{4}$, from which we obtain an estimate of the mass of $\eta^{\prime}$ in $\mathrm{q} \chi \mathrm{PT}$, and the coefficient of quenched chiral logarithm, both in good agreement with the values determined from the pion masses, as well as with the theoretical estimates. With our values of $\delta, A, B$, the experimental inputs of pion and kaon masses, and the pion decay constant, we determine the light quark masses: $m_{u, d}=4.1 \pm 0.3 \mathrm{MeV}$, and $m_{s}=92 \pm 9 \mathrm{MeV}$, in the $\overline{\mathrm{MS}}$ scheme at scale $\mu=2 \mathrm{GeV}$. Also, we determine the quark condensate $\langle\bar{q} q\rangle=-(250 \pm 3 \mathrm{MeV})^{3}$, and the quark-gluon condensate $g\left\langle\bar{q} \sigma_{\mu \nu} F_{\mu \nu} q\right\rangle=-(434 \pm 4 \mathrm{MeV})^{5}$, in the $\overline{\mathrm{MS}}$ scheme at scale $2 \mathrm{GeV}$.

(c) 2003 Elsevier B.V. All rights reserved.
\end{abstract}

PACS: 11.15.Ha; 11.30.Rd; 12.38.Gc; 11.30.Fs

Keywords: Quark mass; Chiral condensate; Quark-gluon condensate; Lattice QCD; Domain-wall quark; Chiral perturbation theory

E-mail address: twchiu@phys.ntu.edu.tw (T.-W. Chiu). 


\section{Introduction}

Exact chiral symmetry on the lattice was pioneered by Kaplan [1] with his proposal of domain-wall fermions (DWF) on the 5-dimensional lattice, in which the fifth dimension (internal flavor space) is discretized with $N_{s}$ sites (flavors) and lattice spacing $a_{5}$. Although the initial motivation was to provide a nonperturbative formulation of chiral gauge theories, the idea turns out to be natural and well-defined for vector-like gauge theories (e.g., QCD), with quark fields constructed from the boundary modes with open boundary conditions [2]. Soon after Kaplan proposed DWF for chiral gauge theories, Narayanan and Neuberger [3] observed that the chiral determinant can be written as the inner-product ("overlap") of two fermionic many body states of two bilinear Hamiltonians. For vector gauge theories like QCD, the fermion determinant is the product of a complex conjugate pair of chiral determinants, thus it is gauge invariant, real, non-negative, and the corresponding lattice Dirac operator for massless quarks (i.e., the overlap Dirac operator [4]) can be represented by a finite matrix of fixed shape regardless of the topology of the background gauge field, without undesired doubling or any fine-tuning. Mathematically, the overlap Dirac operator is exactly equal to the effective 4D lattice Dirac operator (for internal fermions dressed with pseudofermions) of DWF (with $m_{q}=0$ ) in the limit $N_{s} \rightarrow \infty$ followed by $a_{5} \rightarrow 0$,

$$
D=m_{0}\left(1+\gamma_{5} \frac{H_{w}}{\sqrt{H_{w}^{2}}}\right), \quad H_{w}=\gamma_{5} D_{w},
$$

where $D_{w}$ is the standard Wilson-Dirac operator plus a negative parameter $-m_{0}(0<$ $\left.m_{0}<2\right)$.

However, it has been shown [5] that the conventional DWF with any finite $N_{s}$ does not preserve the chiral symmetry optimally for any gauge background.

Recently, one of us (T.W.C.) has proposed a new DWF action $[5,6]$ such that the quark propagator preserves the chiral symmetry optimally for any $N_{s}$ and background gauge field. Further, its effective 4D lattice Dirac operator (with any $N_{s}$ and $m_{q}$ ) for internal fermion loops is exponentially-local for sufficiently smooth gauge backgrounds [7]. In the optimal DWF, the quark fields are constructed from the boundary modes at $s=0$ and $s=N_{s}+1[6]$,

$$
\begin{aligned}
& q(x)=\sqrt{r}\left[P_{-} \psi(x, 0)+P_{+} \psi\left(x, N_{s}+1\right)\right], \quad r=\frac{1}{2 m_{0}}, \\
& \bar{q}(x)=\sqrt{r}\left[\bar{\psi}(x, 0) P_{+}+\bar{\psi}\left(x, N_{s}+1\right) P_{-}\right],
\end{aligned}
$$

and the generating functional for $n$-point Green's function of the quark fields has been derived [6],

$$
Z[J, \bar{J}]=\frac{\int[d U] e^{-\mathcal{A}_{g}} \operatorname{det}\left[\left(D_{c}+m_{q}\right)\left(1+r D_{c}\right)^{-1}\right] \exp \left\{\bar{J}\left(D_{c}+m_{q}\right)^{-1} J\right\}}{\int[d U] e^{-\mathcal{A}_{g}} \operatorname{det}\left[\left(D_{c}+m_{q}\right)\left(1+r D_{c}\right)^{-1}\right]},
$$

where $\mathcal{A}_{g}$ is the action of the gauge fields, $m_{q}$ is the bare quark mass, $\bar{J}$ and $J$ are the Grassman sources of $q$ and $\bar{q}$, respectively, and

$$
r D_{c}=\frac{1+\gamma_{5} S_{\mathrm{opt}}}{1-\gamma_{5} S_{\mathrm{opt}}}
$$




$$
S_{\mathrm{opt}}= \begin{cases}H_{w} R_{Z}^{(n, n)}\left(H_{w}^{2}\right), & N_{s}=2 n+1, \\ H_{w} R_{Z}^{(n-1, n)}\left(H_{w}^{2}\right), & N_{s}=2 n\end{cases}
$$

Here $R_{Z}\left(H_{w}^{2}\right)$ is the Zolotarev optimal rational polynomial [8] for the inverse square root of $H_{w}^{2}$,

$$
\begin{aligned}
R_{Z}^{(n, n)}\left(H_{w}^{2}\right) & =\frac{d_{0}}{\lambda_{\min }} \prod_{l=1}^{n} \frac{1+h_{w}^{2} / c_{2 l}}{1+h_{w}^{2} / c_{2 l-1}} \\
& =\frac{1}{\lambda_{\min }}\left(h_{w}^{2}+c_{2 n}\right) \sum_{l=1}^{n} \frac{b_{l}}{h_{w}^{2}+c_{2 l-1}}, \quad h_{w}^{2}=\frac{H_{w}^{2}}{\lambda_{\min }^{2}}
\end{aligned}
$$

and

$$
R_{Z}^{(n-1, n)}\left(H_{w}^{2}\right)=\frac{d_{0}^{\prime}}{\lambda_{\min }} \frac{\prod_{l=1}^{n-1}\left(1+h_{w}^{2} / c_{2 l}^{\prime}\right)}{\prod_{l=1}^{n}\left(1+h_{w}^{2} / c_{2 l-1}^{\prime}\right)}=\frac{1}{\lambda_{\min }} \sum_{l=1}^{n} \frac{b_{l}^{\prime}}{h_{w}^{2}+c_{2 l-1}^{\prime}},
$$

where the coefficients $d_{0}, d_{0}^{\prime}, c_{l}$ and $c_{l}^{\prime}$ are expressed in terms of elliptic functions [8] with arguments depending only on $N_{s}$ and $\lambda_{\max }^{2} / \lambda_{\min }^{2}\left(\lambda_{\max }\right.$ and $\lambda_{\min }$ are the maximum and the minimum of the eigenvalues of $\left|H_{w}\right|$ ).

From (4), the effective 4D lattice Dirac operator for the fermion determinant is

$$
D\left(m_{q}\right)=\left(D_{c}+m_{q}\right)\left(1+r D_{c}\right)^{-1}=m_{q}+\left(m_{0}-m_{q} / 2\right)\left[1+\gamma_{5} H_{w} R_{Z}\left(H_{w}^{2}\right)\right],
$$

and the quark propagator in background gauge field is

$$
\begin{aligned}
\langle q(x) \bar{q}(y)\rangle & =-\left.\frac{\delta^{2} Z[J, \bar{J}]}{\delta \bar{J}(x) \delta J(y)}\right|_{J=\bar{J}=0} \\
& =\left(D_{c}+m_{q}\right)_{x, y}^{-1}=\left(1-r m_{q}\right)^{-1}\left[D_{x, y}^{-1}\left(m_{q}\right)-r \delta_{x, y}\right] .
\end{aligned}
$$

In practice, we have two ways to evaluate the quark propagator (10) in background gauge field:

(i) To solve the linear system of the 5D optimal DWF operator, as outlined in Ref. [6];

(ii) To solve $D_{x, y}^{-1}\left(m_{q}\right)$ from the system

$$
D\left(m_{q}\right) Y=\left[m_{q}+\left(m_{0}-m_{q} / 2\right)\left(1+\gamma_{5} H_{w} R_{Z}\left(H_{w}^{2}\right)\right)\right] Y=\mathbb{1}
$$

with nested conjugate gradient [9], and then substitute the solution vector $Y$ into (10).

Since either (i) or (ii) yields exactly the same quark propagator, in principle, it does not matter which linear system one actually solves. However, in practice, one should pick the most efficient scheme for one's computational system (hardware and software).

For our present software and hardware (a Linux PC cluster of 42 nodes [10]), it has been geared to the scheme (ii), and it attains the maximum efficiency with Neuberger's 2-pass algorithm [11] for the inner conjugate gradient loop of (11). So, in this paper, we use the scheme (ii) to compute the quark propagator, with the quark fields (2)-(3) defined 
by the boundary modes in the optimal DWF. A study of Neuberger's 2-pass algorithm is presented in Ref. [19].

For our computations in this paper, we fix $m_{0}=1.3$, and project out 20 low-lying eigenmodes and two high-lying modes of $\left|H_{w}\right|$. With $N_{s}=32$, i.e., 16th order Zolotarev rational polynomial $R_{Z}^{(15,16)}\left(H_{w}^{2}\right)$ in (11), we impose stopping criteria $2 \times 10^{-12}$ and $10^{-11}$ for the inner and outer conjugate gradient loops, respectively. Then the chiral symmetry breaking due to finite $N_{s}$ is always less than $10^{-13}$,

$$
\sigma=\left|\frac{Y^{\dagger} S_{\mathrm{opt}}^{2} Y}{Y^{\dagger} Y}-1\right|<10^{-13},
$$

for every iteration of the nested conjugate gradient.

The outline of this paper is as follows. In Section 2, we determine the parameters $(\delta, A, B)$ in the pseudoscalar meson mass formula (14) in quenched chiral perturbation theory ( $\mathrm{q} \chi \mathrm{PT})$ to one-loop order, by computing quenched quark propagators for 13 bare quark masses, and for 100 gauge configurations generated with Wilson gauge action at $\beta=$ 6.0 on the $16^{3} \times 32$ lattice. The pion decay constant is extracted from the pion propagator, from which the lattice spacing is determined to be $a=0.0997(3) \mathrm{fm}=0.5053(15) \mathrm{GeV}^{-1}$. Also, we measure the index (topological) susceptibility of these 100 gauge configurations, $\chi_{t}=(175 \pm 6 \mathrm{MeV})^{4}$, which provides an estimate of $m_{\eta^{\prime}}=813 \pm 51 \mathrm{MeV}$ in $\mathrm{q} \chi \mathrm{PT}$, and the coefficient of quenched chiral logarithm $\delta=0.16(2)$, which is consistent with $\delta=0.164$ (13) determined from the pion masses. In Section 3, with our determined values of $\delta, A, B$, the experimental inputs of pion and kaon masses, and the pion decay constant, we determine the light quark masses: $m_{u, d}=4.1 \pm 0.3 \mathrm{MeV}$, and $m_{s}=92 \pm 9 \mathrm{MeV}$, in the $\overline{\mathrm{MS}}$ scheme at scale $\mu=2 \mathrm{GeV}$. In Section 4 , we determine the quark condensate $\langle\bar{q} q\rangle=$ $-(250 \pm 3 \mathrm{MeV})^{3}$, and the quark-gluon condensate $g\left\langle\bar{q} \sigma_{\mu \nu} F_{\mu \nu} q\right\rangle=-(434 \pm 4 \mathrm{MeV})^{5}$ in the $\overline{\mathrm{MS}}$ scheme at scale $2 \mathrm{GeV}$.

\section{Determination of the parameters in quenched chiral perturbation theory}

In quenched chiral perturbation theory $(\mathrm{q} \chi \mathrm{PT})[13,14]$ the pion mass to one-loop order reads

$$
m_{\pi}^{2}=C m_{q}\left\{1-\delta\left[\ln \left(C m_{q} / \Lambda_{\chi}^{2}\right)+1\right]\right\}+B m_{q}^{2},
$$

where $m_{q}$ denotes the bare ( $u$ and $d$ ) quark mass, $\Lambda_{\chi}$ is the chiral cutoff, $C$ and $B$ are parameters, and $\delta$ is the coefficient of the quenched chiral logarithm. Further, summing all diagrams in which each internal $\eta^{\prime}$ propagator is decorated with any number of "bubble sums" (i.e., summing the leading $\left[\delta \ln \left(C m_{q} / \Lambda_{\chi}^{2}\right)\right]^{n}$ terms to all orders), one obtains [13]

$$
m_{\pi}^{2}=A m_{q}^{\frac{1}{1+\delta}}+B m_{q}^{2}
$$

where

$$
A=\left[C\left(\frac{\Lambda_{\chi}^{2}}{e}\right)^{\delta}\right]^{\frac{1}{1+\delta}} .
$$


Obviously, the value of $\delta$ is independent of the chiral cutoff $\Lambda_{\chi}$ (which is usually taken to be $2 \sqrt{2} \pi f_{\pi}$ with $f_{\pi} \simeq 132 \mathrm{MeV}$ ).

In this section, we determine $\delta$ by fitting our data of $m_{\pi}^{2}$ to (13) and (14), respectively, and find that these two values of $\delta$ are in good agreement with each other.

Theoretically, $\delta$ can be estimated as [13]

$$
\delta=\frac{m_{\eta^{\prime}}^{2}}{8 \pi^{2} f_{\pi}^{2} N_{f}},
$$

where $f_{\pi}$ denotes the pion decay constant, $N_{f}$ denotes the number of light quark flavors, and $m_{\eta^{\prime}}$ denotes the $\eta^{\prime}$ mass in $\mathrm{q} \chi \mathrm{PT}$, which can be estimated as

$$
\sqrt{\mathbf{m}_{\eta^{\prime}}^{2}+m_{\eta}^{2}-2 m_{K}^{2}}=853 \mathrm{MeV}
$$

with experimental values of meson $\operatorname{masses}^{1}: \mathbf{m}_{\eta^{\prime}}=958 \mathrm{MeV}, m_{\eta}=547 \mathrm{MeV}$, and $m_{K}=495 \mathrm{MeV}$. For $f_{\pi}=132 \mathrm{MeV}, N_{f}=3$, and $m_{\eta^{\prime}}=853 \mathrm{MeV}$, (16) gives

$$
\delta \simeq 0.176
$$

Evidently, if one can extract $\delta$ from the data of $m_{\pi}^{2}$, then $m_{\eta^{\prime}}$ in q $\chi$ PT can be determined by (16).

Besides from the data of $m_{\pi}^{2}$, one can also obtain $\delta$ via (16) by extracting $m_{\eta^{\prime}}$ from the propagator of the disconnected hairpin diagram. However, to compute the propagator of the hairpin is very tedious.

Fortunately, with the realization of exact chiral symmetry on the lattice, the quark propagator coupling to $\eta^{\prime}$ is $\left(D_{c}+m_{q}\right)^{-1}$, thus only the zero modes of $D=D_{c}\left(1+r D_{c}\right)^{-1}$ can contribute to the hairpin diagram, regardless of the bare quark mass $m_{q}$. Therefore one can derive an exact relation [15-17] between the $\eta^{\prime}$ mass in $\mathrm{q} \chi \mathrm{PT}$ and the index susceptibility of $D$, without computing the hairpin diagram at all. Explicitly, this exact relation reads as

$$
\left(m_{\eta^{\prime}} a\right)^{2}=\frac{4 N_{f}}{\left(f_{\pi} a\right)^{2}} \frac{\left\langle\left(n_{+}-n_{-}\right)^{2}\right\rangle}{N}=\frac{4 N_{f}}{\left(f_{\pi} a\right)^{2}} \chi_{t},
$$

where $N$ is the total number of sites, and $\chi_{t} \equiv\left\langle\left(n_{+}-n_{-}\right)^{2}\right\rangle / N$ is the index susceptibility of $D$ in the quenched approximation. Then (16) and (19) together gives

$$
\delta=\frac{1}{2 \pi^{2}\left(f_{\pi} a\right)^{4}} \frac{\left\langle\left(n_{+}-n_{-}\right)^{2}\right\rangle}{N} .
$$

A salient feature of (20) is that $\delta$ can be determined at finite lattice spacing $a$, by measuring the index (susceptibility) of $D$, and with $f_{\pi} a$ extracted from the pion propagator.

Now it is clear that, in order to confirm the presence of quenched chiral logarithm in lattice QCD, one needs to check whether the coefficient $\delta$ obtained by fitting (14) to the data of $m_{\pi}^{2}$, agrees with that (20) from the index susceptibility. This is a requirement for the consistency of the theory, since the quenched chiral logarithm in $m_{\pi}^{2}$ (13) is due to the

\footnotetext{
${ }^{1}$ Here we distinguish the physical $\eta^{\prime}$ mass $\left(\mathbf{m}_{\eta^{\prime}}\right)$, from the $\eta^{\prime}$ mass $\left(m_{\eta^{\prime}}\right)$ in q $\chi$ PT.
} 
$\eta^{\prime}$ loop coupling to the pion propagator through the mass term (in the chiral Lagrangian), thus $\delta\left(m_{\eta^{\prime}}\right)$ must be the same in both cases. We regard this consistency requirement as a basic criterion for lattice QCD (with any fermion scheme) to realize QCD chiral dynamics in continuum.

Recently, we have determined $\delta=0.203(14)$, from the data of $m_{\pi}^{2}$, as well as $\delta=$ 0.197 (27) from the index susceptibility, for the $8^{3} \times 24$ lattice at $\beta=5.8$ [15]. Their good agreement suggests that lattice QCD with exact chiral symmetry indeed realizes QCD chiral dynamics in the continuum.

In this paper, we extend our studies to a lattice of larger volume and smaller lattice spacing, $16^{3} \times 32$ at $\beta=6.0$. Details of our computational scheme have been given in Ref. [15], except the inner conjugate gradient loop is now iterated with Neuberger's 2-pass algorithm [11] which not only provides very high precision of chiral symmetry with a fixed amount of memory, but also increases $(\sim 20 \%)$ the speed of our computational system (a Linux PC cluster of 42 nodes at the speed $~ 50$ Gflops [10]). A study of Neuberger's 2-pass algorithm is presented in Ref. [12].

We retrieved 100 gauge configurations from the repository at the Gauge Connection (http://qcd.nersc.gov), which were generated with Wilson gauge action at $\beta=6.0$ on the $16^{3} \times 32$ lattice [18]. For each configuration, quark propagators are computed for 13 bare quark masses.

Then the pion propagator

$$
M_{5}(\vec{x}, t ; \overrightarrow{0}, 0)=\operatorname{tr}\left\{\gamma_{5}\left(D_{c}+m_{q}\right)^{-1}(0, x) \gamma_{5}\left(D_{c}+m_{q}\right)^{-1}(x, 0)\right\}
$$

(where the trace runs over the Dirac and color space), and its time correlation function

$$
G(t)=\sum_{\vec{x}} M_{5}(\vec{x}, t ; \overrightarrow{0}, 0),
$$

are obtained, and $\langle G(t)\rangle$ is fitted by the usual formula

$$
G_{\pi}(t)=\frac{Z}{2 m_{\pi} a}\left[e^{-m_{\pi} a t}+e^{-m_{\pi} a(T-t)}\right]
$$

to extract the pion mass $m_{\pi} a$ and the pion decay constant

$$
f_{\pi} a=2 m_{q} a \frac{\sqrt{Z}}{m_{\pi}^{2} a^{2}} .
$$

In Figs. 1 and 2, we plot the pion mass square $\left(m_{\pi} a\right)^{2}$ and decay constant $f_{\pi} a$ versus bare quark mass $m_{q} a$, respectively.

The data of $f_{\pi} a$ (see Fig. 2) can be fitted by

$$
f_{\pi} a=0.0667(2)+0.2218(20) \times\left(m_{q} a\right)
$$

with $\chi^{2} /$ d.o.f. $=0.95$. Thus taking $f_{\pi} a$ at $m_{q} a=0$ equal to $0.132 \mathrm{GeV}$ times the lattice spacing $a$, we can determine the lattice spacing $a$ and its inverse,

$$
\begin{aligned}
& a^{-1}=\frac{0.132}{f_{0}} \mathrm{GeV}=1.979(6) \mathrm{GeV}, \\
& a=0.0997(3) \mathrm{fm} .
\end{aligned}
$$




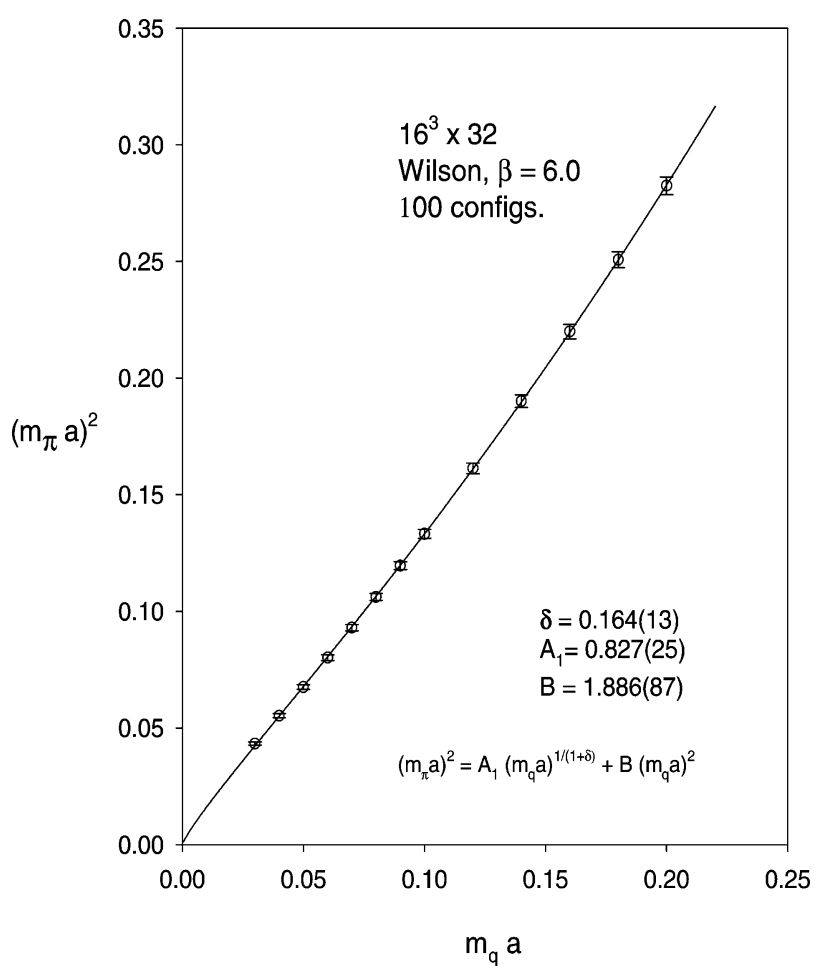

Fig. 1. The pion mass square $\left(m_{\pi} a\right)^{2}$ versus the bare quark mass $m_{q} a$. The solid line is the fit of Eq. (14).

Thus the size of the lattice is $\sim(1.6 \mathrm{fm})^{3} \times 3.2 \mathrm{fm} \simeq(1.9 \mathrm{fm})^{4}$. Since the smallest pion mass is $412 \mathrm{MeV}$, the lattice size is $\sim(3.3)^{3} \times 6.6$, in units of the Compton wavelength $(\sim 0.48 \mathrm{fm})$ of the smallest pion mass. For the largest pion mass $(1052 \mathrm{MeV})$, the lattice spacing is about 0.5 of its Compton wavelength.

Next, rewriting (14) as

$$
m_{\pi}^{2} a^{2}=A_{1}\left(m_{q} a\right)^{\frac{1}{1+\delta}}+B\left(m_{q} a\right)^{2}, \quad A_{1}=A a^{-\frac{1-2 \delta}{1+\delta}}
$$

and fitting to the data of $m_{\pi}^{2}$ (see Fig. 1), we obtain

$$
\begin{aligned}
& \delta=0.164(13), \\
& A_{1}=0.827(25), \\
& B=1.886(87)
\end{aligned}
$$

with $\chi^{2} /$ d.o.f. $=0.54$.

Even though the quenched chiral logarithm may not be easily detected in the graph of $\left(m_{\pi} a\right)^{2}$ versus $m_{q} a$, it can be unveiled by plotting $\left(m_{\pi} a\right)^{2} /\left(m_{q} a\right)$ versus $m_{q} a$, as shown in Fig. 3. Further, plotting $\log \left[\left(m_{\pi} a\right)^{2} /\left(m_{q} a\right)-B\left(m_{q} a\right)\right]$ versus $\log \left(m_{q} a\right)$, the presence of quenched chiral logarithm is evident, as shown in Fig. 4.

Evidently, the coefficient of quenched chiral logarithm $\delta=0.164(13)$ is in good agreement with the theoretical estimate $\delta=0.176$ (Eq. (18)) in q $\chi$ PT. Also, from (16), 


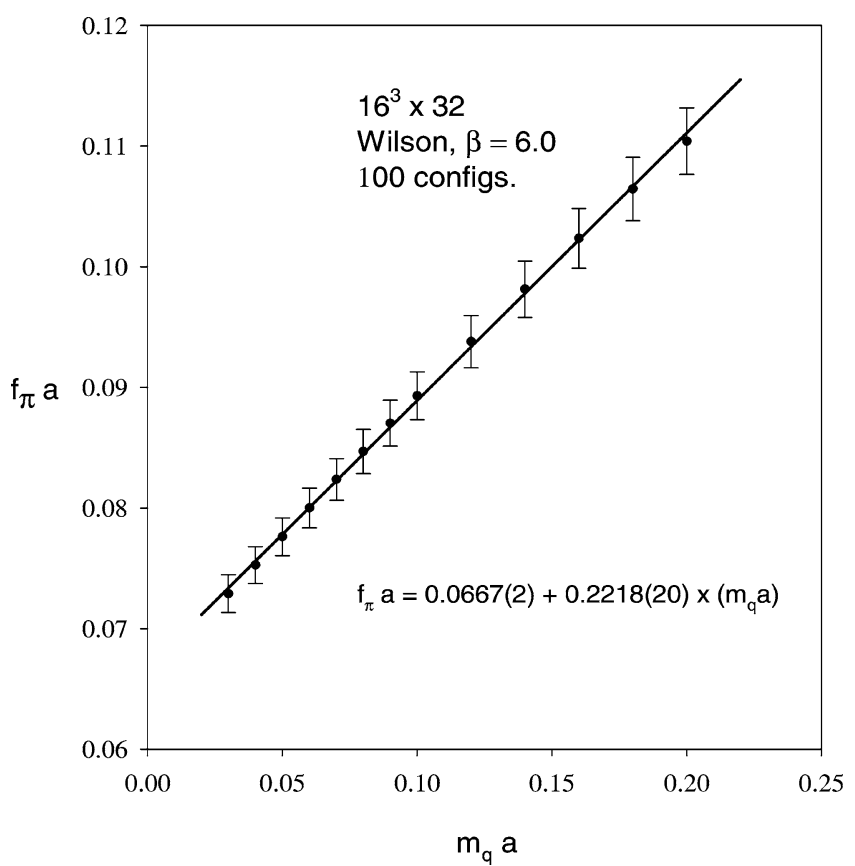

Fig. 2. The pion decay constant $f_{\pi} a$ versus the bare quark mass $m_{q} a$. The solid line is the linear fit.

we obtain an estimate of the $\eta^{\prime}$ mass in $\mathrm{q} \chi \mathrm{PT}$,

$$
m_{\eta^{\prime}}=823 \pm 33 \mathrm{MeV},
$$

which is in good agreement with the theoretical estimate (17).

At this point, it may be instructive to find out what is the value of $\delta$ if one fits (13) to the data of $\left(m_{\pi} a\right)^{2}$, with a fixed $\Lambda_{\chi}$, say $\Lambda_{\chi} a=2 \sqrt{2} \pi f_{\pi} a=2 \sqrt{2} \pi \times 0.0667$. The results (with $\chi^{2} /$ d.o.f. $=0.87$ ) are

$$
\begin{aligned}
& \delta=0.168(17), \\
& C a=1.093(20), \\
& B=2.092(125),
\end{aligned}
$$

where the value of $\delta(28)$ is in good agreement with (24), and other parameters are also consistent with those determined with (14).

Note that, in Fig. 3, the minimum of $\left(m_{\pi} a\right)^{2} /\left(m_{q} a\right)$ occurs at $m_{q} a \simeq 0.09$, which corresponds to $m_{q} \simeq 180 \mathrm{MeV}$ for $a \simeq 0.0997 \mathrm{fm}$. This is consistent with the data of $10^{3} \times 24$, and $12^{3} \times 24$ lattices at $\beta=5.8$ [19], where the minima of $\left(m_{\pi} a\right)^{2} /\left(m_{q} a\right)$ occur at $m_{q} \simeq 190$ and $217 \mathrm{MeV}$, respectively. That is, the minima of $\left(m_{\pi} a\right)^{2} /\left(m_{q} a\right)$ for these three lattices occur in the range of $m_{q} \simeq 200 \pm 20 \mathrm{MeV}$. This suggests that one should be able to observe the quenched chiral logarithm behavior (in the plot of $\left(m_{\pi} a\right)^{2} /\left(m_{q} a\right)$ versus $m_{q} a$ ) for $m_{q} \leqslant 200 \mathrm{MeV}$ (i.e., $m_{\pi} \leqslant 600 \mathrm{MeV}$ ). 


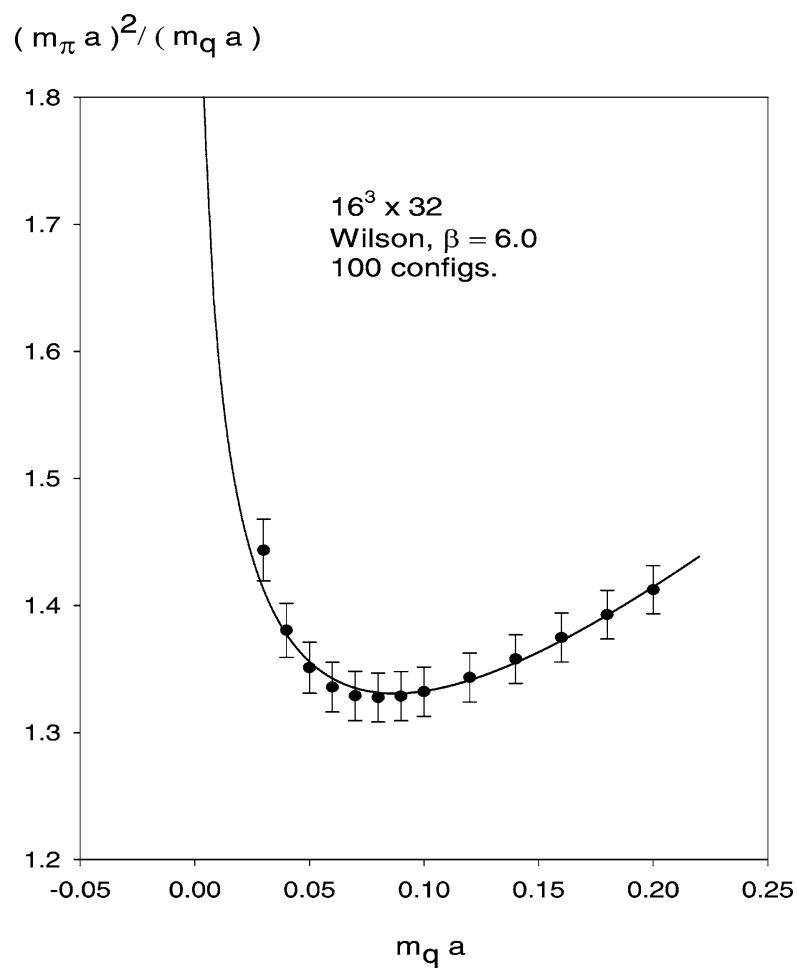

Fig. 3. $\left(m_{\pi} a\right)^{2} /\left(m_{q} a\right)$ versus the bare quark mass $m_{q} a$.

However, in a recent study of chiral logs in quenched lattice QCD with Iwasaki gauge action [20], the location of the minimum of $\left(m_{\pi} a\right)^{2} /\left(m_{q} a\right)$ occurs at $m_{q} a \simeq 0.13$ with $a \simeq 0.2 \mathrm{fm}$, which corresponds to $m_{q} \simeq 130 \mathrm{MeV}$ (i.e., $m_{\pi} \simeq 480 \mathrm{MeV}$ ). This seems to suggest that the location of the minimum of $\left(m_{\pi} a\right)^{2} /\left(m_{q} a\right)$ depends on the gauge action as well as the lattice spacing $a$. For a recent review of quenched chiral logarithms of various quark actions and gauge actions, see Refs. [21,22] and the references therein.

In order to provide a self-consistency check of the coefficient of quenched chiral $\operatorname{logarithm}(\delta)$ determined from the data of $m_{\pi}^{2}$, we measure the index susceptibility of $D$ by the spectral flow method, and then determine $\delta$ via (20).

The distribution of the indices of $D$ for these 100 gauge configurations is listed in Table 1. So the index (topological) susceptibility is

$$
\begin{aligned}
& a^{4} \chi_{t}=\frac{\left\langle\left(n_{+}-n_{-}\right)^{2}\right\rangle}{N}=6.03(75) \times 10^{-5}, \\
& \chi_{t}=(175 \pm 6 \mathrm{MeV})^{4},
\end{aligned}
$$

where $N$ is the total number of sites. 


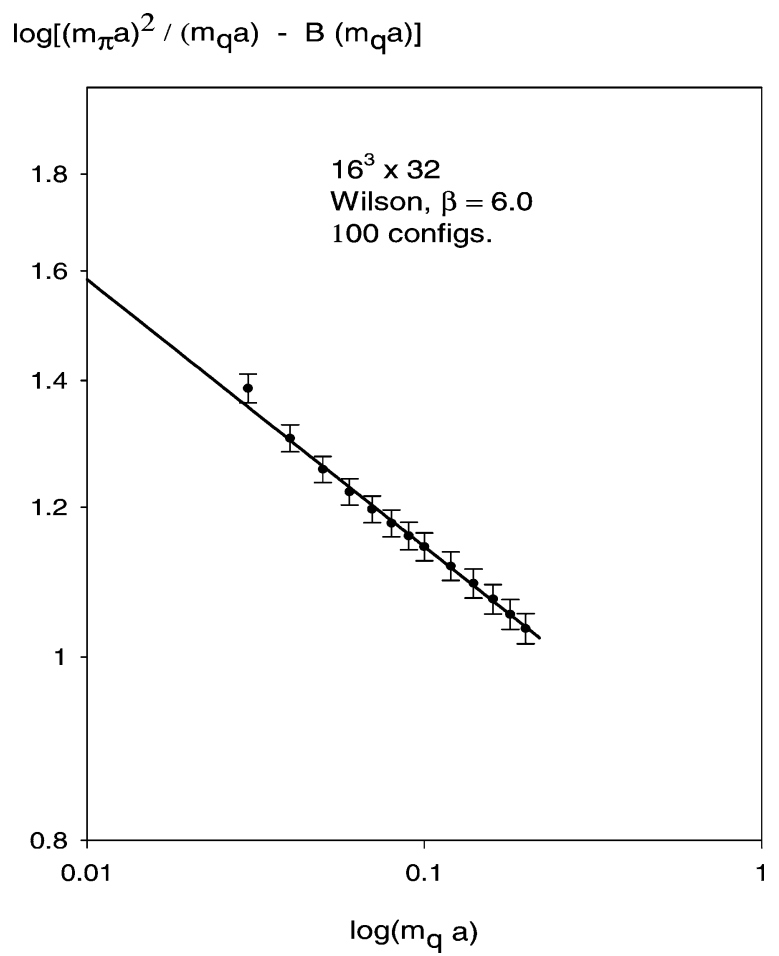

Fig. 4. The extraction of the quenched chiral logarithm by plotting $\log \left[\left(m_{\pi} a\right)^{2} /\left(m_{q} a\right)-B\left(m_{q} a\right)\right]$ versus $\log \left(m_{q} a\right)$. The slope of the fitted straight line is equal to $-\delta /(1+\delta)$.

Table 1

The distribution of the indices of $D$ for 100 gauge configurations at $\beta=6.0$ on the $16^{3} \times 32$ lattice

\begin{tabular}{cc}
\hline$n_{+}-n_{-}$ & Number of configurations \\
\hline 5 & 6 \\
4 & 5 \\
3 & 6 \\
2 & 8 \\
1 & 14 \\
0 & 10 \\
-1 & 15 \\
-2 & 15 \\
-3 & 10 \\
-4 & 4 \\
-5 & 3 \\
-6 & 3 \\
-7 & 1 \\
\hline
\end{tabular}


Now substituting the index susceptibility (31), the lattice spacing (23), $f_{\pi}=132 \mathrm{MeV}$, and $N_{f}=3$, into the exact relation (19), we obtain the $\eta^{\prime}$ mass

$$
m_{\eta^{\prime}}=813 \pm 51 \mathrm{MeV},
$$

which agrees with the theoretical estimate (17). Next we substitute (31) and $f_{\pi} a=$ $0.0667(2)$ into (20), and get

$$
\delta=0.16 \pm 0.02,
$$

which is in good agreement with the value (28) determined from the data of $m_{\pi}^{2}$, as well as with the theoretical estimate (18).

Note that a finite lattice must impose a lower bound for the pion mass, as well as the corresponding one for the bare quark mass. If one decreases the bare quark mass beyond its lower bound, then the resulting pion mass would be flattening (or increasing) rather than decreasing. This is essentially due to the finite volume effects of the zero modes of the lattice Dirac operator, which is proportional to $\left|n_{+}-n_{-}\right| /\left(m_{q}^{2} a^{2} N\right)$ as $m_{q} a \rightarrow 0$. Thus, only in the infinite volume limit $(N \rightarrow \infty)$, one can obtain zero pion mass with zero bare quark mass. Nevertheless, for a finite lattice, the finite volume effects of the zero modes can be suppressed if the bare quark mass $m_{q} a \gg \sqrt{\left|n_{+}-n_{-}\right| / N}$. For a lattice of size $16^{3} \times 32$ at $\beta=6.0$, we find that $m_{q} a \geqslant 0.03$ is sufficient to suppress the finite volume effects of the zero modes. This can be verified by extracting the "pion" mass $m_{4} a$ from the correlation function of the "pion" propagator with $\gamma_{4} \gamma_{5}$ coupling

$$
\operatorname{tr}\left[\gamma_{4} \gamma_{5}\left(D_{c}+m_{q}\right)^{-1}(0, x) \gamma_{4} \gamma_{5}\left(D_{c}+m_{q}\right)^{-1}(x, 0)\right]
$$

which is void of the contributions of topological zero modes, and then compare $m_{4} a$ with $m_{\pi} a$. In Fig. 5, $m_{4} a$ is plotted against $m_{\pi} a$, for the same $m_{q} a$. The good agreement between $m_{4} a$ and $m_{\pi} a$ for each of the 13 bare quark masses provides an estimate that the error due to the finite volume effect of the zero modes is less than $5 \%$, thus is well under control.

In passing, we present our data of the vector meson mass. In Fig. 6, we plot $m_{\rho} a$ versus $m_{\pi} a$. At the smallest bare quark mass $m_{q} a=0.03$ in our data, the ratio $m_{\pi} / m_{\rho}$ is $0.435(8)$. Fitting our data to the vector meson mass formula [23] in $\mathrm{q} \chi \mathrm{PT}$,

$$
m_{\rho} a=C_{0}+\delta C_{1 / 2}\left(m_{\pi} a\right)+C_{1}\left(m_{\pi} a\right)^{2}+C_{3 / 2}\left(m_{\pi} a\right)^{3},
$$

we obtain

$$
\begin{aligned}
& C_{0}=0.474(6), \\
& \delta C_{1 / 2}=-0.223(56), \\
& C_{1}=1.370(158), \\
& C_{3 / 2}=-0.542(141)
\end{aligned}
$$

with $\chi^{2} /$ d.o.f. $=0.92$.

On the other hand, if we plot $m_{\rho} a$ versus $\left(m_{\pi} a\right)^{2}$, as shown in Fig. 7 , then the data seems to be well fitted by

$$
m_{\rho} a=0.495(4)+4.57(23) \times\left(m_{\pi} a\right)^{2}
$$




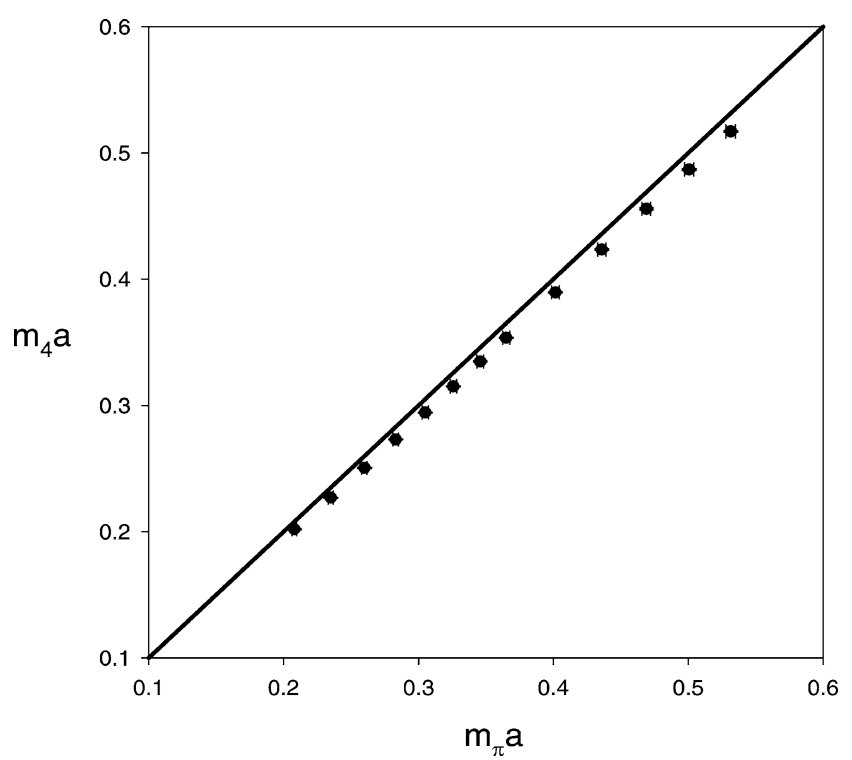

Fig. 5. The "pion" mass $m_{4} a$ versus the pion mass $m_{\pi} a$. The solid line is $m_{4}=m_{\pi}$.

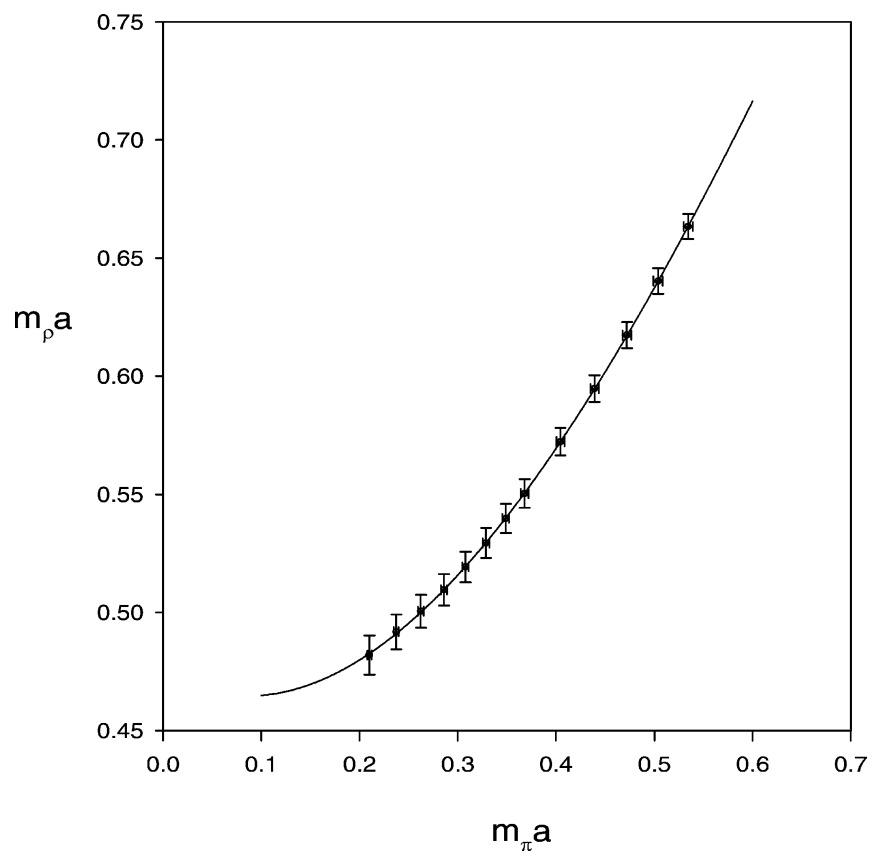

Fig. 6. The vector meson mass $m_{\rho} a$ versus the pion mass $m_{\pi} a$ for 13 bare quark masses. The solid line is the fit to Eq. (35). 


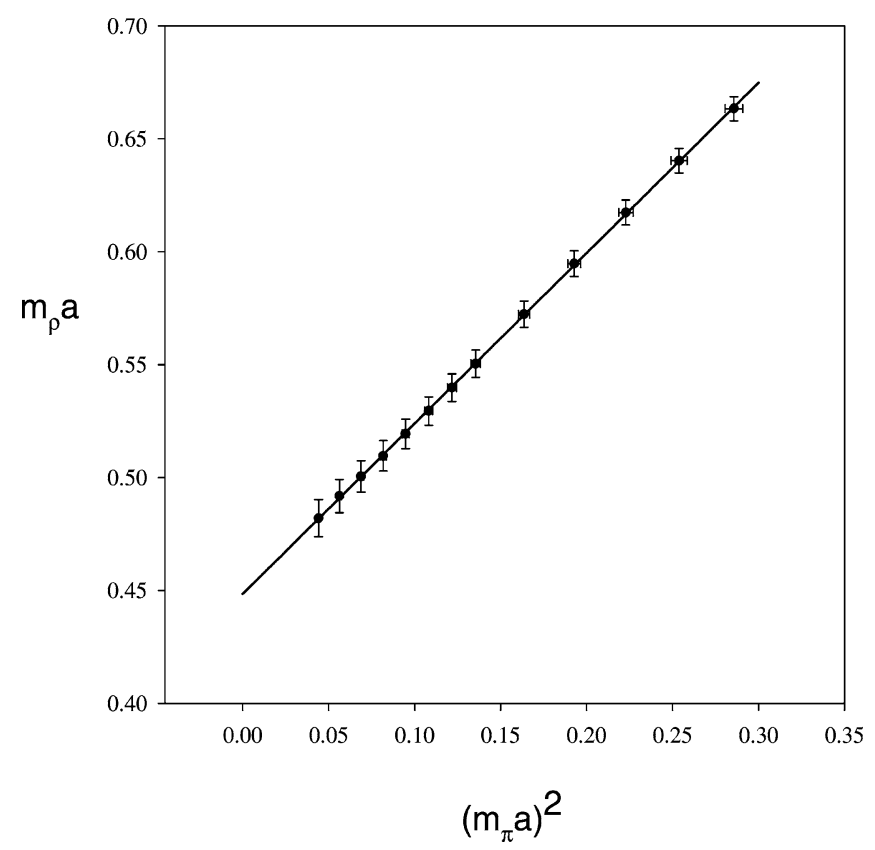

Fig. 7. The vector meson mass $m_{\rho} a$ versus the pion mass square $\left(m_{\pi} a\right)^{2}$ for 13 bare quark masses. The solid line is the linear fit, Eq. (36).

with $\chi^{2} /$ d.o.f. $=0.98$.

With our present data, it seems to be unlikely to rule out either one of above two possibilities. We hope to return to this problem elsewhere.

Note that even though one has no technical difficulties to use smaller quark masses to push $m_{\pi} / m_{\rho}$ down to smaller values, say, 0.2 , one suspects that the finite size effects (especially for $m_{\pi}$ ) might be too large to be meaningful. Further, for consistency, all quantities in this paper are determined with the same set of quark propagators computed for 13 bare quark masses ranging from $m_{q} a=0.03$ to 0.2 .

\section{Light quark masses}

In the standard model, the quark masses are fundamental parameters which have to be determined from high energy experiments. However, they cannot be measured directly since quarks are confined inside hadrons, unlike an isolated electron whose mass and charge both can be measured directly from its responses in electric and magnetic fields. Therefore, the quark masses can only be determined by comparing a theoretical calculation of physical observables (e.g., hadron masses) with the experimental values. Evidently, for any field theoretic calculation, the quark masses depend on the regularization, as well as the renormalization scheme and scale.

One of the objectives of lattice QCD is to compute the hadron masses nonperturbatively from the first principle, and from which the quark masses are determined. However, the 
performance of the present generation of computers is still quite remote from what is required for computing the light hadron masses at the physical (e.g., $m_{\pi} \simeq 140 \mathrm{MeV}$ ) scale, on a lattice with enough sites in each direction such that the discretization errors as well as the finite volume effects are both negligible comparing to the statistical ones. Nevertheless, even with lattices of moderate sizes, lattice QCD can determine the values of the parameters in the hadron mass formulas of the (quenched) chiral perturbation theory. Then one can use these formulas to evaluate the hadron masses at the physical scale, as well as to determine the quark masses.

In quenched chiral perturbation theory $[13,14]$, the ratio of pion and kaon masses to one-loop order reads

$$
\frac{m_{K}^{2}}{m_{\pi}^{2}}=\frac{m+m_{s}}{2 m}\left\{1+\delta\left[1-\frac{m}{m_{s}-m} \ln \left(\frac{m_{s}}{m}\right)\right]\right\},
$$

where $m$ denotes $u$ and $d$ quark bare masses in the isospin limit $\left(m_{u}=m_{d} \equiv m\right), m_{s}$ the $s$ quark bare mass, $\delta$ the coefficient of the quenched chiral logarithm.

With experimental values of pion and kaon masses as inputs to (38) and (37), one can determine the ratio of light quark bare masses $m_{s} / m$, but the absolute scale cannot be fixed by quenched chiral perturbation theory. At this point, lattice QCD plays the important role to fix the values of the parameters $A, B$ and $\delta$ in the pion mass formula (14),

$$
m_{\pi}^{2}=A m^{\frac{1}{1+\delta}}+B m^{2},
$$

by measuring the pion mass versus the bare quark mass. Then the light quark masses $m$ and $m_{s}$ can be determined with experimental inputs of $m_{\pi}, m_{K}$, and $f_{\pi}$ (to fix the lattice spacing $a$ ).

In Section 2, we have determined the lattice spacing $a$, and the parameters $\delta, A_{1}$ and $B$ (23)-(26), by computing the quenched quark propagators for 100 gauge configurations generated with the Wilson gauge action at $\beta=6.0$ on the $16^{3} \times 32$ lattice. Now inserting $\delta$ (28) and experimental values of meson masses $\left(m_{K}=495 \mathrm{MeV}\right.$ and $\left.m_{\pi}=135 \mathrm{MeV}\right)$ into Eq. (37), we obtain the quark mass ratio

$$
\frac{m_{s}}{m}=22.58(23)
$$

comparing with the ratio at the zeroth order $(\delta=0)$

$$
\left(\frac{m_{s}}{m}\right)_{0}=25.89
$$

From (38), with values of $a, \delta, A_{1}$ and $B$ in (23)-(26), and $m_{\pi}=135 \mathrm{MeV}$, we obtain

$$
m=4.7 \pm 0.5 \mathrm{MeV},
$$

which is inserted into (39) to yield

$$
m_{s}=107 \pm 11 \mathrm{MeV}
$$

In order to transcribe above results (41)-(42) to their corresponding values in the usual renormalization scheme $\overline{\mathrm{MS}}$ in high energy phenomenology, one needs to compute the lattice renormalization constant $Z_{m}=Z_{s}^{-1}$, where $Z_{s}$ is the renormalization constant for 
$\bar{\psi} \psi$. In general, $Z_{m}$ should be determined nonperturbatively. In this paper, we use the one loop perturbation formula [24]

$$
Z_{s}=1+\frac{g^{2}}{4 \pi^{2}}\left[\ln \left(a^{2} \mu^{2}\right)+6.7722\right] \quad\left(m_{0}=1.30\right),
$$

to obtain an estimate of $Z_{s}$. At $\beta=6.0, a=0.0997(3) \mathrm{fm}$, and $\mu=2 \mathrm{GeV}$, (43) gives $Z_{s}=1.172(1)$. Thus the quark masses in (41)-(42) are transcribed to

$$
\begin{aligned}
& m_{u, d}^{\overline{\mathrm{MS}}}(2 \mathrm{GeV})=4.1 \pm 0.3 \pm 1.0 \mathrm{MeV}, \\
& m_{s}^{\overline{\mathrm{MS}}}(2 \mathrm{GeV})=92 \pm 9 \pm 16 \mathrm{MeV},
\end{aligned}
$$

where the first errors are statistical errors, and the second errors are our estimate of systematic errors due to finite volume and discretization effects, by comparing our present results to our earlier ones [25] with smaller volume and larger lattice spacing. Evidently, the light quark masses in (44), (45) are in good agreement with the current lattice world average [22], as well as the strange quark mass determined in Ref. [26].

\section{Chiral condensate and quark-gluon condensate}

\subsection{Chiral condensate}

The chiral condensate in lattice QCD with the optimal DWF can be expressed as

$$
\begin{aligned}
-\langle\bar{q} q\rangle & =\left.\lim _{m_{q} \rightarrow 0} \lim _{\Omega \rightarrow \infty} \frac{1}{\Omega} \sum_{x} \frac{\delta}{\delta J(x)} \frac{\delta}{\delta \bar{J}(x)} Z[J, \bar{J}]\right|_{J=\bar{J}=0} \\
& =\lim _{m_{q} \rightarrow 0 \Omega \rightarrow \infty} \lim _{\Omega} \frac{1}{\Omega} \frac{\int[d U] e^{-\mathcal{A}_{g}} \operatorname{det} D\left(m_{q}\right) \sum_{x} \operatorname{tr}\left(D_{c}+m_{q}\right)_{x, x}^{-1}}{\int[d U] e^{-\mathcal{A}_{g}} \operatorname{det} D\left(m_{q}\right)} \\
& \equiv \Sigma\left(N_{f}\right),
\end{aligned}
$$

where $\Omega=N a^{4}$ is the volume of the 4D lattice, $N_{f}$ is the number of quark flavors, $Z[J, \bar{J}]$ is the generating functional in (4), and the Dirac, color, and flavor indices have been suppressed.

The subtlety of (46) lies in its two limiting processes. If the order of these two limiting processes is exchanged, then $\langle\bar{q} q\rangle$ must be zero since spontaneous chiral symmetry could not occur on a finite lattice for exactly massless fermions. Thus it seems to be unlikely to be able to extract the chiral condensate of QCD on a finite lattice, not to mention in the quenched approximation. ${ }^{2}$ Nevertheless, using chiral perturbation theory or random matrix theory, the chiral condensate at finite volume and quark mass can be computed in the continuum, both for unquenched and quenched QCD. In the quenched approximation, the chiral condensate with fixed $m_{q}, \Omega$, and $Q$ (topological charge)

\footnotetext{
${ }^{2}$ Recall that the $\eta^{\prime}$ loop in the quark propagator leads to the quenched chiral logarithm in the chiral condensate.
} 


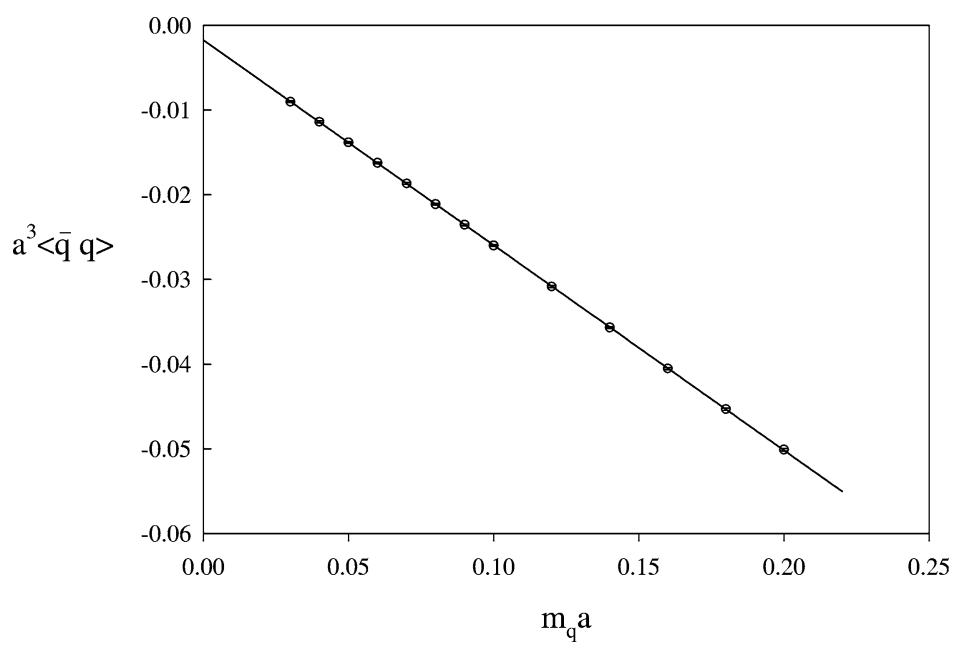

Fig. 8. The chiral condensate $a^{3}\langle\bar{q} q\rangle$ versus the bare quark mass $m_{q} a$. The solid line is the linear fit (49).

reads [27]

$$
-\langle\bar{q} q\rangle_{m, \Omega, Q}=\Sigma\left\{\frac{|Q|}{z}+z\left[I_{|Q|}(z) K_{|Q|}(z)+I_{|Q|+1}(z) K_{|Q|-1}(z)\right]\right\},
$$

where $\Sigma=\Sigma\left(N_{f}=0\right), z=m_{q} \Omega \Sigma$, and $I_{Q}$ and $K_{Q}$ are modified Bessel functions. Thus, the chiral condensate can be extracted by measuring $\langle\bar{q} q\rangle$ in different topological sectors at different $m_{q}$ and $\Omega$. Alternatively, a plausible way to extract the chiral condensate on a finite lattice (with large volume $\Omega$ ) is to measure $\operatorname{tr}\left(D_{c}+m_{q}\right)_{x, x}^{-1}$, i.e.,

$$
-\langle\bar{q} q\rangle=\frac{\int[d U] e^{-\mathcal{A}_{g}} \operatorname{tr}\left(D_{c}+m_{q}\right)_{x, x}^{-1}}{\int[d U] e^{-\mathcal{A}_{g}}},
$$

for a set of small quark masses $m_{q}$ satisfying $z \gg 1$. Then the chiral condensate at $m_{q}=0$ can be obtained by linear extrapolation, since $\operatorname{tr}\left(D_{c}+m_{q}\right)_{x, x}^{-1}$ behaves like a linear function of $m_{q}$ for small $m_{q}$. This ansatz can be verified in practice.

In the following, we measure $\langle\bar{q} q\rangle$ in the quenched approximation, with one fixed site at the origin $(\overrightarrow{0}, 0)$, for 100 gauge configurations generated with Wilson gauge action at $\beta=6.0$ on the $16^{3} \times 32$ lattice. The results are plotted in Fig. 8 for 13 bare quark masses, and they are very well fitted by the straight line

$$
-a^{3}\langle\bar{q} q\rangle=1.73(3) \times 10^{-3}+0.242(0) \times\left(m_{q} a\right) .
$$

Now we take the value $1.73(3) \times 10^{-3}$ at $m_{q} a=0$ to be the chiral condensate, then we obtain

$$
\langle\bar{q} q\rangle=-0.0134(2) \mathrm{GeV}^{3},
$$


with the inverse lattice spacing $a^{-1}$ (23) determined in Section 2. Using the one-loop renormalization $Z_{s}$ (43), we transcribe the chiral condensate (50) to $\overline{\mathrm{MS}}$ at scale $\mu=$ $2 \mathrm{GeV}$,

$$
\langle\bar{q} q\rangle^{\overline{\mathrm{MS}}}(2 \mathrm{GeV})=-(250 \pm 3 \mathrm{MeV})^{3},
$$

which is in good agreement with the phenomenological estimate, as well as other recent lattice results [26,28,29]. Nevertheless, a complete picture of the underlying mechanism of spontaneous symmetry breaking in QCD can only be unveiled in the context of dynamical quarks.

\subsection{Quark-gluon condensate}

The quark-gluon condensate $g\left\langle\bar{q} \sigma_{\mu \nu} F_{\mu \nu} q\right\rangle$ is one of the nonperturbative features of the QCD vacuum. It is one of the parameters in the framework of QCD sum rule to determine the low-energy properties of QCD [30,31].

The first measurement [32] of the quark-gluon condensate in lattice QCD was performed more than 15 years ago, with the staggered fermion, for only 5 gauge configurations. A recent measurement [33] was also carried out with the staggered fermion, at each site along the body diagonal of the $16^{4}$ lattice, for 100 gauge configurations generated with Wilson gauge action at $\beta=6.0$. In Ref. [33], with 1600 measurements for each quark mass (of total 3 quark masses), the quark condensate and quarkgluon condensate at $m_{q}=0$ were obtained by linear extrapolation, however, the ratio $g\left\langle\bar{q} \sigma_{\mu \nu} F_{\mu \nu} q\right\rangle /\langle\bar{q} q\rangle$ turns out to be much larger than the value in the QCD sum rule as well as other phenomenological estimates.

In general, the quark-gluon condensate in lattice QCD with the optimal DWF can be expressed as

$$
\begin{aligned}
- & g\left\langle\bar{q} \sigma_{\mu \nu} F_{\mu \nu} q\right\rangle \\
& =\left.\lim _{m_{q} \rightarrow 0} \lim _{\Omega \rightarrow \infty} \frac{1}{\Omega} \sum_{x} \frac{\delta}{\delta J(x)} \sigma_{\mu \nu} F_{\mu \nu}(x) \frac{\delta}{\delta \bar{J}(x)} Z[J, \bar{J}]\right|_{J=\bar{J}=0} \\
& =\lim _{m_{q} \rightarrow 0} \lim _{\Omega \rightarrow \infty} \frac{1}{\Omega} \frac{\int[d U] e^{-\mathcal{A}_{g}} \operatorname{det} D\left(m_{q}\right) \sum_{x} \operatorname{tr}\left[\left(D_{c}+m_{q}\right)_{x, x}^{-1} \sigma_{\mu \nu} F_{\mu \nu}(x)\right]}{\int[d U] e^{-\mathcal{A}_{g}} \operatorname{det} D\left(m_{q}\right)},
\end{aligned}
$$

where $\Omega=(N a)^{4}$ is the volume of the 4D lattice, $Z[J, \bar{J}]$ is the generating functional in (4), and the Dirac, color, and flavor indices have been suppressed. Here the matrix valued field tensor $F_{\mu \nu}(x)$ can be obtained from the four plaquettes surrounding $x$ on the $(\hat{\mu}, \hat{v})$ plane, i.e.,

$$
\begin{aligned}
g a^{2} F_{\mu \nu}(x) \simeq \frac{1}{8 i}[ & P_{\mu \nu}(x)+P_{\mu \nu}(x-\hat{\mu})+P_{\mu \nu}(x-\hat{v})+P_{\mu \nu}(x-\hat{\mu}-\hat{v}) \\
& \left.\quad-P_{\mu \nu}^{\dagger}(x)-P_{\mu \nu}^{\dagger}(x-\hat{\mu})-P_{\mu \nu}^{\dagger}(x-\hat{v})-P_{\mu \nu}^{\dagger}(x-\hat{\mu}-\hat{v})\right],
\end{aligned}
$$

where

$$
P_{\mu \nu}(x)=U_{\mu}(x) U_{v}(x+\hat{\mu}) U_{\mu}^{\dagger}(x+\hat{v}) U_{\nu}^{\dagger}(x) .
$$




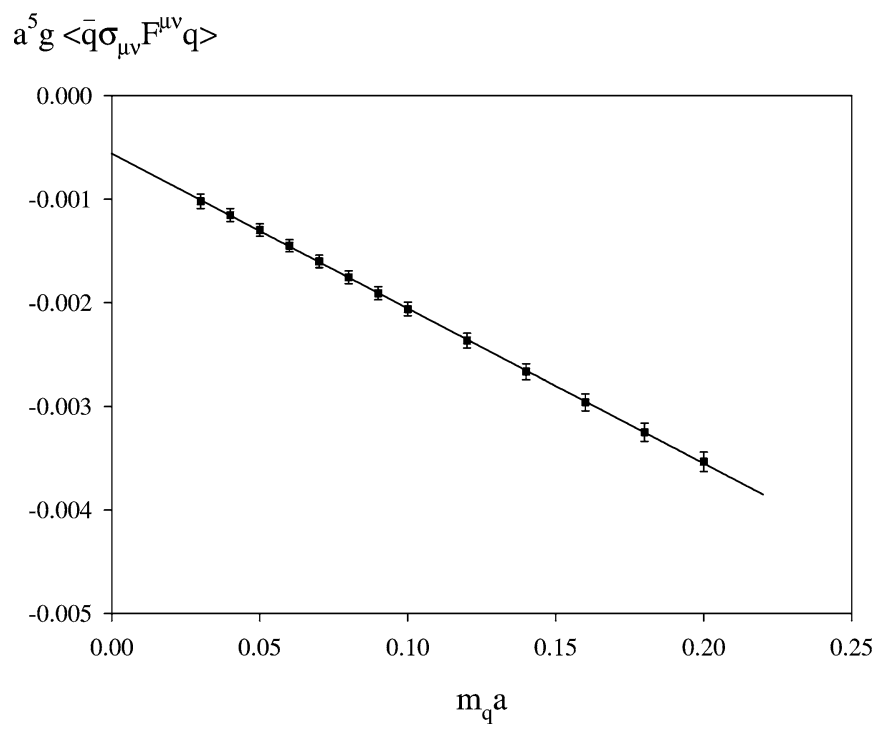

Fig. 9. The quark-gluon condensate $a^{5} g\left\langle\bar{q} \sigma_{\mu \nu} F_{\mu \nu} q\right\rangle$ versus the bare quark mass $m_{q} a$. The solid line is the linear fit.

In the following, we measure the quark-gluon condensate in quenched approximation, with one fixed site at the origin $(\overrightarrow{0}, 0)$, for 100 gauge configurations generated with Wilson gauge action at $\beta=6.0$ on the $16^{3} \times 32$ lattice. The results are plotted in Fig. 9 for 13 bare quark masses, and they are well fitted by the straight line

$$
-a^{5} g\left\langle\bar{q} \sigma_{\mu \nu} F_{\mu \nu} q\right\rangle=5.59(12) \times 10^{-4}+0.0150(0) \times\left(m_{q} a\right) .
$$

Now we take the value $5.59(12) \times 10^{-4}$ at $m_{q} a=0$ to be the quark-gluon condensate, then we obtain

$$
g\left\langle\bar{q} \sigma_{\mu \nu} F_{\mu \nu} q\right\rangle=-0.0170(4) \mathrm{GeV}^{5}
$$

with the inverse lattice spacing $a^{-1}$ (23) determined in Section 2. Using the one loop renormalization [24], we transcribe the quark-gluon condensate (54) to $\overline{\mathrm{MS}}$ at scale $\mu=2 \mathrm{GeV}$,

$$
g\left\langle\bar{q} \sigma_{\mu \nu} F_{\mu \nu} q\right\rangle^{\overline{\mathrm{MS}}}(2 \mathrm{GeV})=-(434 \pm 4 \mathrm{MeV})^{5} .
$$

Thus the ratio of the quark-gluon condensate (55) to the quark condensate (51) is

$$
M_{0}^{2}=\left.\frac{g\left\langle\bar{q} \sigma_{\mu \nu} F_{\mu \nu} q\right\rangle}{\langle\bar{q} q\rangle}\right|^{\overline{\mathrm{MS}}}(2 \mathrm{GeV})=0.98(2) \mathrm{GeV}^{2}
$$

which becomes $0.92(2) \mathrm{GeV}^{2}$ at $\mu=0.5 \mathrm{GeV}$, in agreement with the estimate $0.8 \pm$ $0.2 \mathrm{GeV}^{2}[34]$ in the QCD sum rule. 


\section{Summary and discussions}

In this paper, we have determined several quantities (the topological susceptibility (31), the parameters $\delta, A_{1}, B(23)-(30)$ in the pseudoscalar meson mass formulas, the light quark masses (44), (45), the quark condensate (51), and the quark-gluon condensate (55)) in quenched lattice QCD with exact chiral symmetry, with quark fields defined by the boundary modes (2)-(3) of the optimal DWF. The propagator of the 4D effective Dirac operator, $D^{-1}\left(m_{q}\right),(11)$ is solved with Neuberger's 2-pass algorithm [11], for 100 gauge configurations [18] generated by Wilson gauge action at $\beta=6.0$ on the $16^{3} \times 32$ lattice. Our results provide strong evidence that lattice QCD with exact chiral symmetry indeed realizes the quenched QCD chiral dynamics.

The coefficient of quenched chiral logarithm $(\delta=0.164 \pm 0.013)$ extracted from the pion mass agrees very well with that $(\delta=0.16 \pm 0.02)$ obtained from the topological susceptibility. The agreement provides a consistency check of the theory. Also, both of them are in good agreement with the theoretical estimate $\delta \simeq 0.176$ in quenched chiral perturbation theory.

Even though the size of our lattice is insufficient for computing the light hadron masses at the physical (e.g., $m_{\pi} \simeq 140 \mathrm{MeV}$ ) scale, we can determine the values of the parameters $\delta, A_{1}, B(23)-(30)$ in the pseudoscalar meson mass formulas of $\mathrm{q} \chi \mathrm{PT}$, and then use these formulas to evaluate the pseudoscalar masses at the physical scale, as well as to determine the light quark masses $m_{u, d}$ and $m_{s},(44),(45)$.

Using the Gell-Mann-Oakes-Renner relation,

$$
m_{\pi}^{2}=-\frac{4 m\langle\bar{\psi} \psi\rangle}{f_{\pi}^{2}},
$$

and Eqs. (37) and (13), we can estimate the chiral condensate at the zeroth order

$$
\begin{aligned}
& \langle\bar{u} u\rangle_{0}=-\frac{C f_{\pi}^{2}}{4}=-0.00942(18) \mathrm{GeV}^{3}, \\
& \langle\bar{s} s\rangle_{0}=\langle\bar{u} u\rangle_{0} \frac{m}{m_{s}}\left(\frac{2 m_{K}^{2}}{m_{\pi}^{2}}-1\right)=-0.0108(3) \mathrm{GeV}^{3},
\end{aligned}
$$

where (29), (23), (39), and the experimental inputs $\left(m_{\pi}=135 \mathrm{MeV}, m_{K}=495 \mathrm{MeV}\right)$ have been used. Using one-loop renormalization (43), we transcribe (57) to $\overline{\mathrm{MS}}$ at scale $\mu=2 \mathrm{GeV}$,

$$
\begin{aligned}
& \langle\bar{u} u\rangle_{0}^{\overline{\mathrm{MS}}}(2 \mathrm{GeV})=-(223 \pm 3 \mathrm{MeV})^{3}, \\
& \langle\bar{s} s\rangle_{0}^{\overline{\mathrm{MS}}}(2 \mathrm{GeV})=-(233 \pm 6 \mathrm{MeV})^{3} .
\end{aligned}
$$

It is expected that the values of the quark condensates (59)-(60) are different from (51), since the formers are zeroth order estimates using the pseudoscalar mass formulas with parameters determined by the long distance behaviors of the pion propagator, while the latter is determined by the short distance behaviors of the quark propagator.

The quark-gluon condensate as well as the chiral condensate are the basic features of the QCD vacuum. In the framework of the QCD sum rules, they are introduced as parameters in the nonperturbative generalization of operator product expansion, and are related to 
hadronic properties. Therefore, it is important for lattice QCD to determine these quantities from the first principles. Our determination of the quark-gluon condensate (55) is the first result of this quantity from lattice QCD with exact chiral symmetry. The agreement of the ratio (of the quark-gluon condensate to the chiral condensate), $M_{0}^{2} \overline{\mathrm{MS}}(0.5 \mathrm{GeV})=$ $0.92(2) \mathrm{GeV}^{2}$, with the estimate $0.8(2) \mathrm{GeV}^{2}$ in the QCD sum rule is encouraging, which suggests that lattice QCD with exact chiral symmetry does provide a viable framework to tackle the low energy physics of QCD pertaining to the nonperturbative QCD vacuum.

\section{Acknowledgements}

T.W.C. would like to thank Steve Sharpe for helpful discussions and suggesting (14). This work was supported in part by the National Science Council, ROC, under the grant number NSC91-2112-M002-025.

\section{References}

[1] D.B. Kaplan, Phys. Lett. B 288 (1992) 342;

D.B. Kaplan, Nucl. Phys. B (Proc. Suppl.) 30 (1993) 597.

[2] Y. Shamir, Nucl. Phys. B 406 (1993) 90; V. Furman, Y. Shamir, Nucl. Phys. B 439 (1995) 54.

[3] R. Narayanan, H. Neuberger, Phys. Lett. B 302 (1993) 62;

R. Narayanan, H. Neuberger, Nucl. Phys. B 412 (1994) 574;

R. Narayanan, H. Neuberger, Phys. Rev. Lett. 71 (1993) 3251.

[4] H. Neuberger, Phys. Lett. B 417 (1998) 141.

[5] T.W. Chiu, Phys. Rev. Lett. 90 (2003) 071601.

[6] T.W. Chiu, hep-lat/0303008.

[7] T.W. Chiu, Phys. Lett. B 552 (2003) 97.

[8] N.I. Akhiezer, Theory of Approximation, Dover, New York, 1992;

N.I. Akhiezer, Elements of the Theory of Elliptic Functions, in: Translations of Mathematical Monographs, Vol. 79, American Mathematical Society, Providence, RI, 1990.

[9] H. Neuberger, Phys. Rev. Lett. 81 (1998) 4060.

[10] T.W. Chiu, T.H. Hsieh, C.H. Huang, T.R. Huang, hep-lat/0208039, Int. J. Mod. Phys. C (2003), in press.

[11] H. Neuberger, Int. J. Mod. Phys. C 10 (1999) 1051.

[12] T.W. Chiu, T.H. Hsieh, hep-lat/0306025.

[13] S.R. Sharpe, Phys. Rev. D 46 (1992) 3146.

[14] C.W. Bernard, M.F. Golterman, Phys. Rev. D 46 (1992) 853.

[15] T.W. Chiu, T.H. Hsieh, Phys. Rev. D 66 (2002) 014506.

[16] T. DeGrand, U.M. Heller, Phys. Rev. D 65 (2002) 114501.

[17] L. Giusti, G.C. Rossi, M. Testa, G. Veneziano, Nucl. Phys. B 628 (2002) 234.

[18] We retrieved the first 100 gauge configurations of OSU-Q60a produced by G. Kilcup, D. Pekurovsky, L. Venkataraman, Nucl. Phys. B (Proc. Suppl.) 53 (1997) 345, from the repository at the Gauge Connection http://qcd.nersc.gov/.

[19] T.W. Chiu, T.H. Hsieh, Nucl. Phys. B (Proc. Suppl.) 119C (2003) 793.

[20] S.J. Dong, T. Draper, I. Horvath, F.X. Lee, K.F. Liu, N. Mathur, J.B. Zhang, hep-lat/0304005.

[21] L. Giusti, Nucl. Phys. B (Proc. Suppl.) 119 (2002) 149.

[22] H. Wittig, Nucl. Phys. B (Proc. Suppl.) 119 (2002) 59.

[23] M. Booth, G. Chiladze, A.F. Falk, Phys. Rev. D 55 (1997) 3092.

[24] C. Alexandrou, H. Panagopoulos, E. Vicari, Nucl. Phys. B 571 (2000) 257;

C. Alexandrou, E. Follana, H. Panagopoulos, E. Vicari, Nucl. Phys. B 580 (2000) 394. 
[25] T.W. Chiu, T.H. Hsieh, Phys. Lett. B 538 (2002) 298.

[26] L. Giusti, C. Hoelbling, C. Rebbi, Phys. Rev. D 64 (2001) 114508.

[27] J.C. Osborn, D. Toublan, J.J. Verbaarschot, Nucl. Phys. B 540 (1999) 317;

P.H. Damgaard, J.C. Osborn, D. Toublan, J.J. Verbaarschot, Nucl. Phys. B 547 (1999) 305.

[28] P. Hasenfratz, S. Hauswirth, T. Jorg, F. Niedermayer, K. Holland, Nucl. Phys. B 643 (2002) 280.

[29] P. Hernandez, K. Jansen, L. Lellouch, H. Wittig, Nucl. Phys. B (Proc. Suppl.) 106 (2002) 766.

[30] M.A. Shifman, A.I. Vainshtein, V.I. Zakharov, Nucl. Phys. B 147 (1979) 385;

M.A. Shifman, A.I. Vainshtein, V.I. Zakharov, Nucl. Phys. B 147 (1979) 448;

M.A. Shifman, A.I. Vainshtein, V.I. Zakharov, Nucl. Phys. B 147 (1979) 519.

[31] B.L. Ioffe, Nucl. Phys. B 188 (1981) 317;

B.L. Ioffe, Nucl. Phys. B 191 (1981) 591, Erratum.

[32] M. Kremer, G. Schierholz, Phys. Lett. B 194 (1987) 283.

[33] T. Doi, N. Ishii, M. Oka, H. Suganuma, Phys. Rev. D 67 (2003) 054504.

[34] V.M. Belyaev, B.L. Ioffe, Sov. Phys. JETP 56 (1982) 493, Zh. Eksp. Teor. Fiz. 83 (1982) 876 (in Russian). 